\title{
ANÁLISIS Y DISEÑO DE MEJORA DE DESEMPEÑO ESTRUCTURAL DE PÉRGOLAS
}

\author{
Analysis and design of improvement of \\ structural performance of pergolas
}

\section{EPISTEMUS}

ISSN: 2007-8196 (electrónico)

ISSN: 2007-4530 (impresa)

Gema Karina Ibarra Torúa ${ }^{1}$

David Ruiz Aguirre ${ }^{2}$

Dagoberto Burgos Flores 3

Ilse Irene Yepis Osuna 4

Recibido: 12 de septiembre de 2016,

Aceptado: 30 de noviembre del 2016

Autor de Correspondencia:

Dra. Gema Karina Ibarra Torúa

Correo: gema.ibarra@unison.mx

\section{Resumen}

Este articulo expone la problemática de un sistema estructural de soporte para viñedos llamado pérgola, el cual se busca tenga un mejor desempeño estructural con respecto a la sección actual. Éste, lleva a cabo la revisión de capacidades estructurales mediante ensayes experimentales, modelos numéricos de barras y con elementos solidos bajo el rango elástico lineal, teniendo como finalidad el análisis y diseño de una propuesta de sección transversal de larguero.

Palabras clave: Desempeño Estructural, diseño estructural, sección transversal.

\section{Abstract}

This paper exposes the problematic of a structural support system for vineyards called pérgola, with this is sought to have a better structural performance with respect to the current section. This one, carries out the revision of structural capacities by means of experimental tests, numerical models of bars and with solid elements under the linear elastic rank, having like purpose the analysis and design of a proposal of stringer beam cross section.

Keywords: Structural Performance, structural design, cross section.

1 Universidad de Sonora. Correo: gema.ibarra@unison.mx 


\section{INTRODUCCIÓN}

El estudio presentado se elabora teniendo como finalidad el análisis y diseño de una propuesta de sección transversal de larguero (perfil estructural de pared delgada) elemento componente del sistema estructural de soporte para viñedos Ilamado pérgola el cual se busca tenga un mejor desempeño estructural con respecto a la sección actual, esto bajo las mismas condiciones de apoyo y geométricas globales. Este, lleva a cabo la revisión de capacidades estructurales mediante ensayes experimentales, modelos numéricos de barras y con elementos solidos bajo el rango elástico lineal. Para garantizar la correcta simulación de las cargas externas a las que se somete el perfil de manera cotidiana se analiza bajo cargas gravitacionales y laterales criticas (por ejemplo, viento).

El sistema estructural de soporte (pérgola) en estudio está compuesto por los siguientes elementos:

1. Larguero (izquierdo y derecho).

2. Cruceta,

3. Tutor o poste

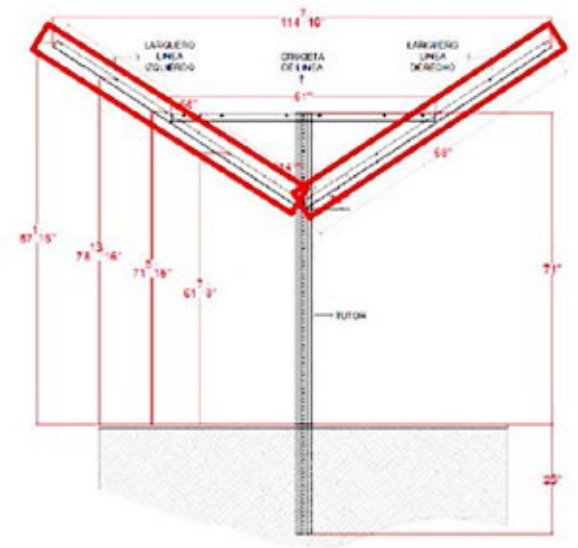

Imagen 1. Pergola tipo

Tal como se puede observar se compone de un poste empotrado en el suelo al cual se unen los elementos principales de carga y objeto de estudio de este documento, los largueros, los cuales por cuestiones de estabilidad están unidos al poste y entre sí mediante un elemento llamado cruceta.

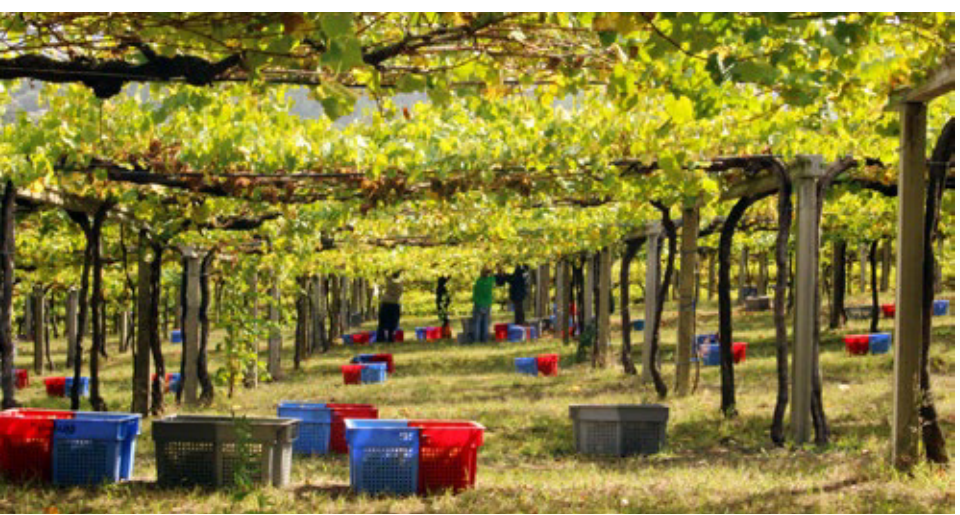

\section{Pérgolas de línea 61" x 68"}

Larguero línea derecha y línea izquierda: Longitud $68^{\prime \prime}$. Angulo de $33^{\circ}$ con respecto a la horizontal; $114^{\circ}$ entre larguero izquierdo y derecho. Distancia de extremo del larguero izquierdo y derecho $1147 " / 16$. Cruceta de línea: Longitud61". Tutor: Longitud 96". 25" de empotramiento.

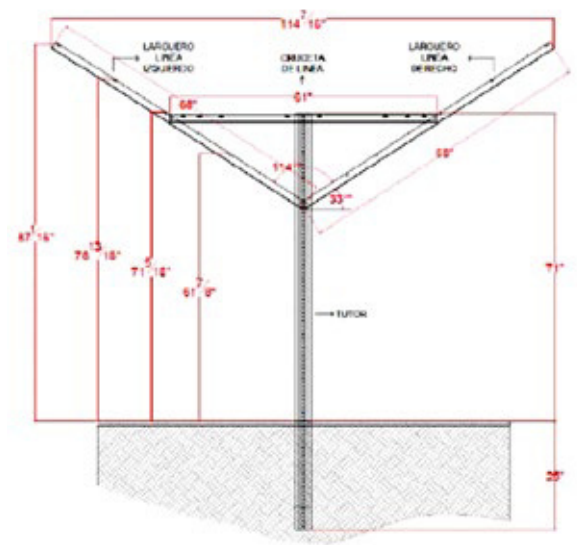

Imagen 1.1. Pergola 61" x 68"

\section{Pérgolas de línea 36" x 60"}

Larguero línea derecha y línea izquierda: Longitud 60 ". Angulo de $25^{\circ}$ con respecto a la horizontal. $131^{\circ}$ entre larguero izquierdo y derecho. Distancia de extremo del larguero izquierdo y derecho 109 7/16". Cruceta de línea: Longitud 36". Tutor: Longitud 96", 25" de empotramiento.

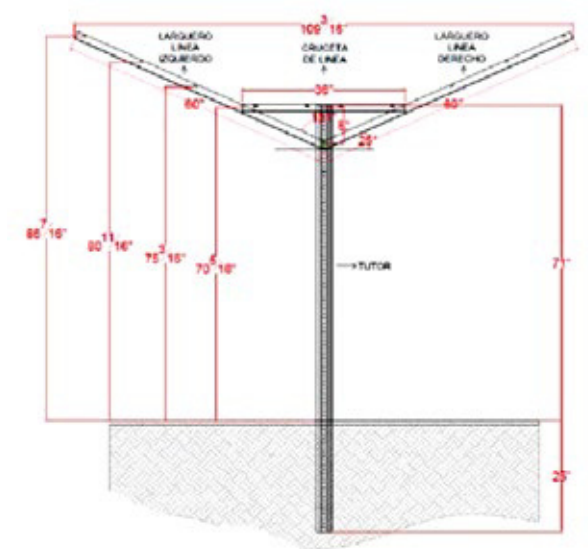

Imagen 1.2. Pérgola 36" x 60"

\section{OBJETIVO}

Diseñar una propuesta de sección transversal de larguero, elemento componente del sistema estructural de soporte para viñedos llamado pérgola el cual se busca tenga un mejor desempeño estructural con respecto a la sección actual. 


\section{PLANTEAMIENTO DEL PROBLEMA}

El estudio realizado surge ante la inquietud de un cliente, al presentar un problema en un sistema estructural de soporte de viñedos, llamado pérgola, el cual no cumplía con las especificaciones y capacidades necesarias para soportar el sistema de cargas que lo regían.

\section{METODO DE TRABAJO}

Como parte de este estudio se realiza una comparativa de tres diferentes diseños de larguero, todos con un desarrollo o ancho de placa de $2 \frac{1}{2}{ }^{\prime \prime}$, los cuales se describen a continuación:

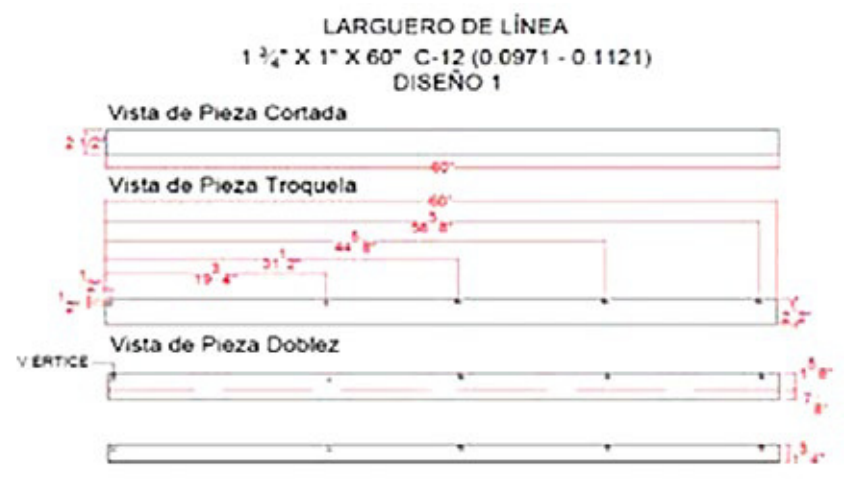

Imagen 2. Diseño de Larguero tipo.

En general el larguero tiene las siguientes dimensiones, sin embargo, el cambio será en su sección transversal.

DISEÑO A, el cual se propone para mejorar la capacidad de carga en el eje fuerte del perfil, sin embargo, se observa una falla prematura por pandeo lateral (eje débil).
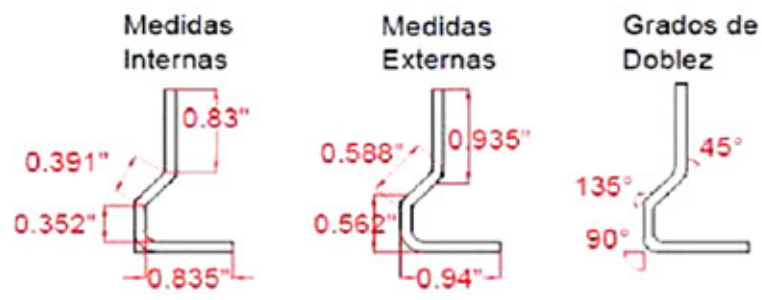

Imagen 2.1. Diseño de Larguero " $A$ ".

¡DISEÑO L, sección transversal de uso común la cual se pretende mejorar mediante los cambios en su geometría.

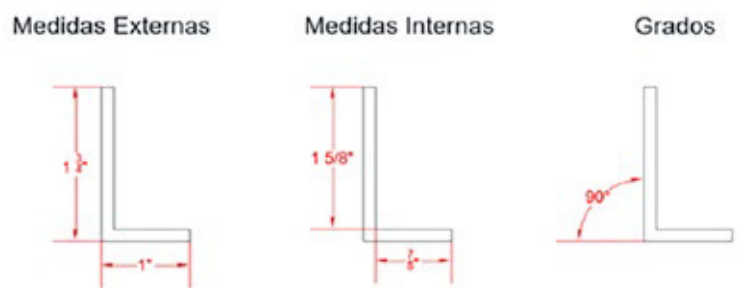

Imagen 2.2. Diseño de Larguero “ $L$ ”.
DISEÑO A", el cual se propone para mejorar la capacidad de carga en el eje fuerte del perfil, con aumento en su capacidad de carga lateral (eje débil), esto mediante un labio atiesador en el patín.

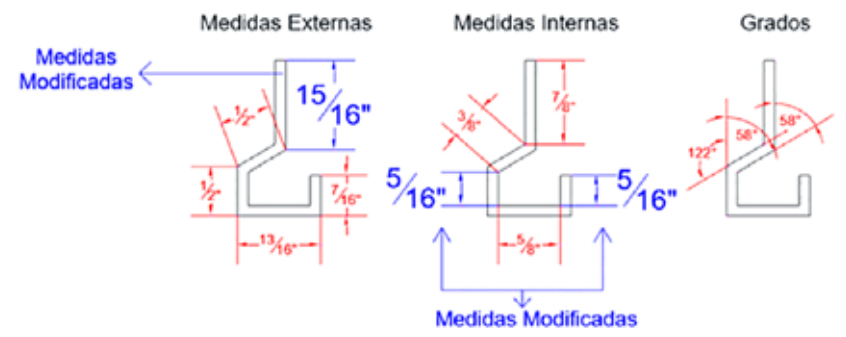

Imagen 2.3. Diseño de Larguero " $\mathrm{A}$ ".

\section{iANÁLISIS}

El análisis general de la estructura será elástico usando métodos matriciales.

Se toman en cuenta en el análisis las actividades que llevan a la determinación de la respuesta de la estructura entre las diferentes acciones exteriores que pueden afectarla.

La respuesta de la estructura está representada por el conjunto de parámetros físicos que describen su comportamiento ante las acciones que le son aplicadas.

Para que el sistema estructural cumpla con las funciones para las cuales está siendo diseñado, es necesario que la respuesta de la estructura se mantenga dentro de los límites que no afecten su correcto funcionamiento ni su estabilidad. Para tomar en cuenta todos estos aspectos se requiere de lo siguiente:

a) Modelar la estructura. Idealizar la estructura real por medio de un modelo teórico factible de ser analizado con los procedimientos de cálculo disponibles.

1) Modelo de Barras (MEF)

2) Modelo de Solidos (MEF)

* Validados por ensayes experimentales de tensión y flexión.

b) Determinar las acciones de diseño. Particularmente para este estudio las acciones de diseño no están determinadas por códigos o reglamentos, por otro lado, las cargas (gravitacionales y laterales) definidas se toman como aquellas que conducen a un estado límite de falla al elemento estructural.

c) Determinar los efectos de las acciones. En esta etapa, que constituye el análisis propiamente dicho, se determinan las fuerzas internas (momentos flexionantes y de torsión, fuerzas axiales, cortantes, esfuerzos, etc.) así como las flechas y deformaciones de la misma. Los métodos 
de análisis suponen en general un comportamiento elástico lineal.

\section{PROGRAMAS DE DISEÑO}

Para realizar el análisis y revisión de las propuestas se utilizó el software SOLIDWORKS (Modelos solidos) y SAP2000 V16 (Modelos de barras), la mayor parte del análisis y revisión se realizó por medio de la modelación numérica en estos softwares, por tanto, nos referiremos constantemente al modelo y a sus gráficas para justificar los resultados, todo esto con la debida validación mediante los ensayes experimentales.

\section{CARGAS}

Las cargas aplicadas a los modelos numéricos obedecen a las cargas a la falla obtenidas en los ensayes experimentales, así como a las resistencias nominales obtenidas analíticamente mediante las expresiones para resistencia a flexión del ANSI/AISC 360-10. El nivel de cargas es variable de acuerdo al tipo de modelo, pero sobre todo a las condiciones de apoyo del elemento en estudio, por lo que se mostraran niveles de carga distintos manteniendo valores similares cuando las condiciones antes mencionadas sean similares, la razón del por qué se consideraron distintas condiciones de apoyo se explicará en el siguiente capítulo.

A continuación, se muestran las cargas aplicadas en los modelos y ensayes experimentales.

Batería de cargas monótonas (hasta llevar a la falla) correspondientes a los ensayes experimentales. Cabe señalar que todos los especímenes se cargaron hasta la falla con la misma velocidad de aplicación de carga.

Para evaluar la capacidad de los distintos perfiles de mejora y de línea, las cargas se aplicaron en los planos ortogonales de pandeo (eje fuerte y débil del perfil), añadiendo la variable de las perforaciones en la sección en tres tipos barrenada, muesca y sin perforación.

Tabla 1. Primera batería de pruebas (40 elementos ensayados) a perfiles a flexión con apoyo simple con carga puntual al centro.

CORRIDA 1

\begin{tabular}{|c|c|c|c|c|c|c|}
\hline \multirow{2}{*}{ Perfil } & \multirow{2}{*}{$\begin{array}{c}\text { Modo de } \\
\text { ensaye }\end{array}$} & \multicolumn{3}{|c|}{ Espesor (mm) } & \multirow{2}{*}{$\begin{array}{l}\text { Tipo de } \\
\text { Hueco }\end{array}$} & \multirow{2}{*}{$\begin{array}{c}\text { Carga Final } \\
\text { (kgf) }\end{array}$} \\
\hline & & A & B & C & & \\
\hline$A^{\prime \prime}$ & V & 2.74 & 2.72 & 2.71 & $M$ & 580 \\
\hline$A^{\prime \prime}$ & V & 2.71 & 2.75 & 2.7 & B & 692 \\
\hline$A^{\prime \prime}$ & $\mathrm{H}$ & 2.72 & 2.74 & 2.72 & B & 432 \\
\hline$A^{\prime \prime}$ & $\mathrm{H}$ & 2.74 & 2.71 & 2.74 & $M$ & 420 \\
\hline L & V & 2.74 & 2.71 & 2.73 & $M$ & 540 \\
\hline A & V & 2.73 & 2.71 & 2.71 & $M$ & 532 \\
\hline A & V & 2.68 & 2.69 & 2.7 & B & 744 \\
\hline L & V & 2.78 & & 2.78 & B & 732 \\
\hline L & V & 2.76 & & 2.76 & L & 989 \\
\hline A & $\mathrm{H}$ & 2.74 & 2.74 & 2.74 & $M$ & 308 \\
\hline L & $\mathrm{H}$ & 2.76 & & 2.74 & L & 292 \\
\hline L & $\mathrm{H}$ & 2.77 & & 2.74 & $M$ & 304 \\
\hline A & $\mathrm{H}$ & 2.72 & & 2.71 & L & 388 \\
\hline A & $\mathrm{H}$ & 2.72 & & 2.72 & B & 348 \\
\hline A & V & 2.72 & & 2.74 & $\mathrm{~L}$ & 828 \\
\hline L & $\mathrm{H}$ & 2.75 & & 2.76 & B & 368 \\
\hline \multicolumn{7}{|c|}{$\begin{array}{l}\mathbf{V}=\text { Vertical (Eje fuerte, posición comun de carga del perfil) } \\
\mathbf{H}=\text { Horizontal (Eje debil, posición normal a cargas laterales) }\end{array}$} \\
\hline \multicolumn{2}{|c|}{$\mathbf{M}=$ Muesca } & \multicolumn{3}{|c|}{$\mathbf{B}=$ Barreno } & in perfor & \\
\hline
\end{tabular}


Tabla 2. Segunda batería de pruebas ( 14 elementos ensayados) a perfiles a flexión con apoyo simple con carga puntual al centro.

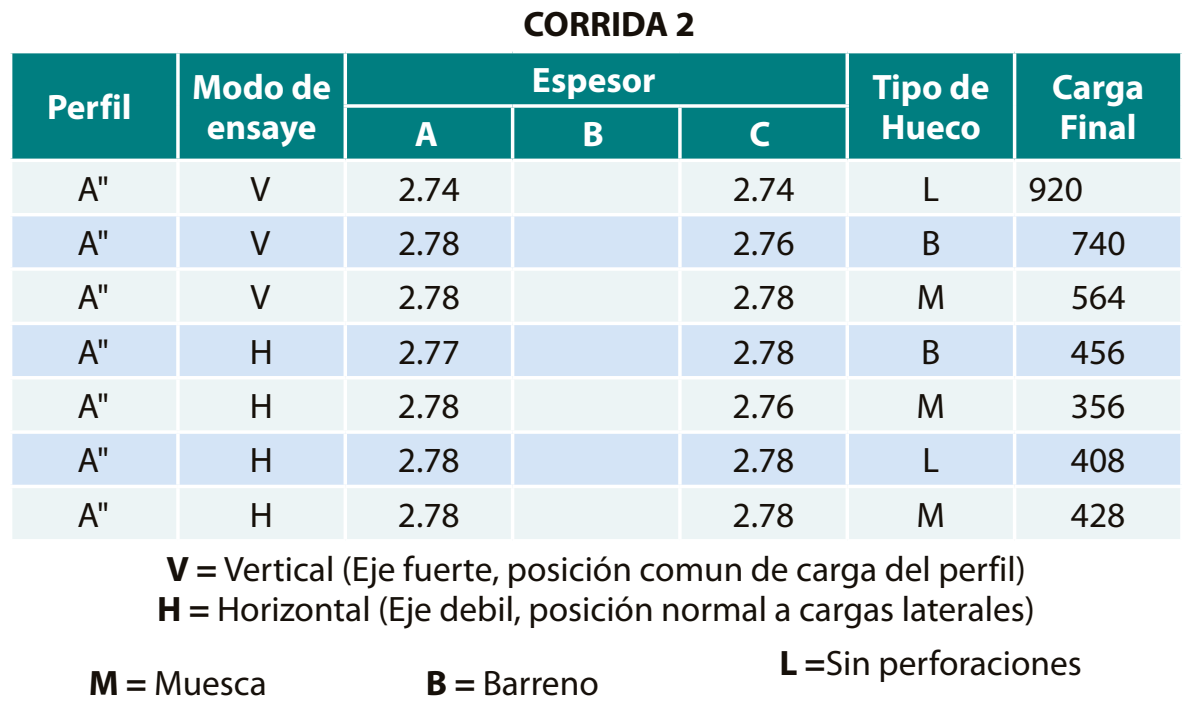

A continuación, se muestran las cargas aplicadas en los modelos sólidos.

\section{Liso Con Carga En Cara Vertical}

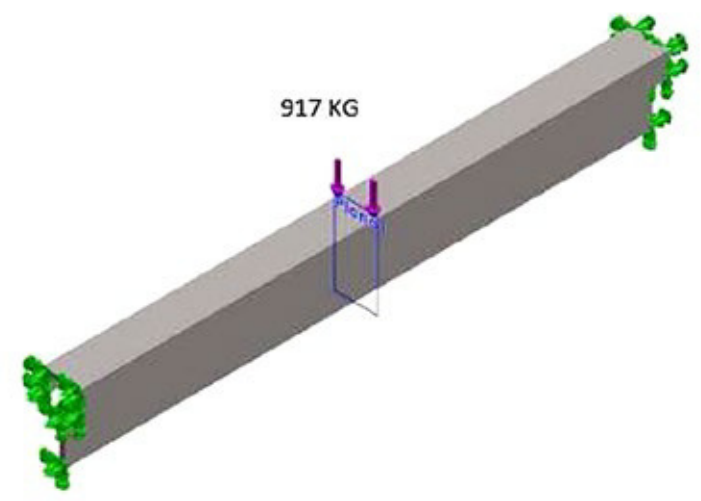

Imagen 3. Carga aplicada en el sentido del eje fuerte para elementos con carga al centro y apoyo simple, esto con la intención de validar con condiciones de ensayes experimentales.

Tabla 3. Valor típico de carga en el sentido del eje fuerte de la sección.

\begin{tabular}{|c|c|c|}
\hline Nombre de carga & Cargar imagen & Detalles de carga \\
\hline Fuerza-3 & till & $\begin{array}{c}\text { Entidades: } 1 \text { cara(s) } \\
\text { Tipo: Aplicar fuerza } \\
\text { normal Valor: } 9000 \text { N }\end{array}$ \\
\hline
\end{tabular}

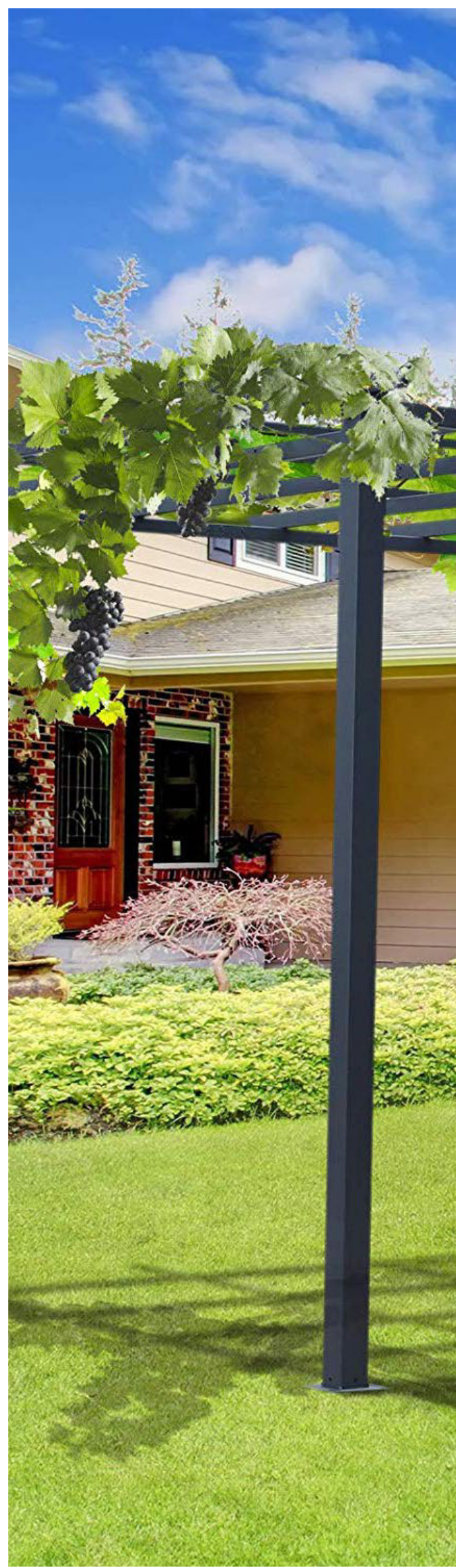



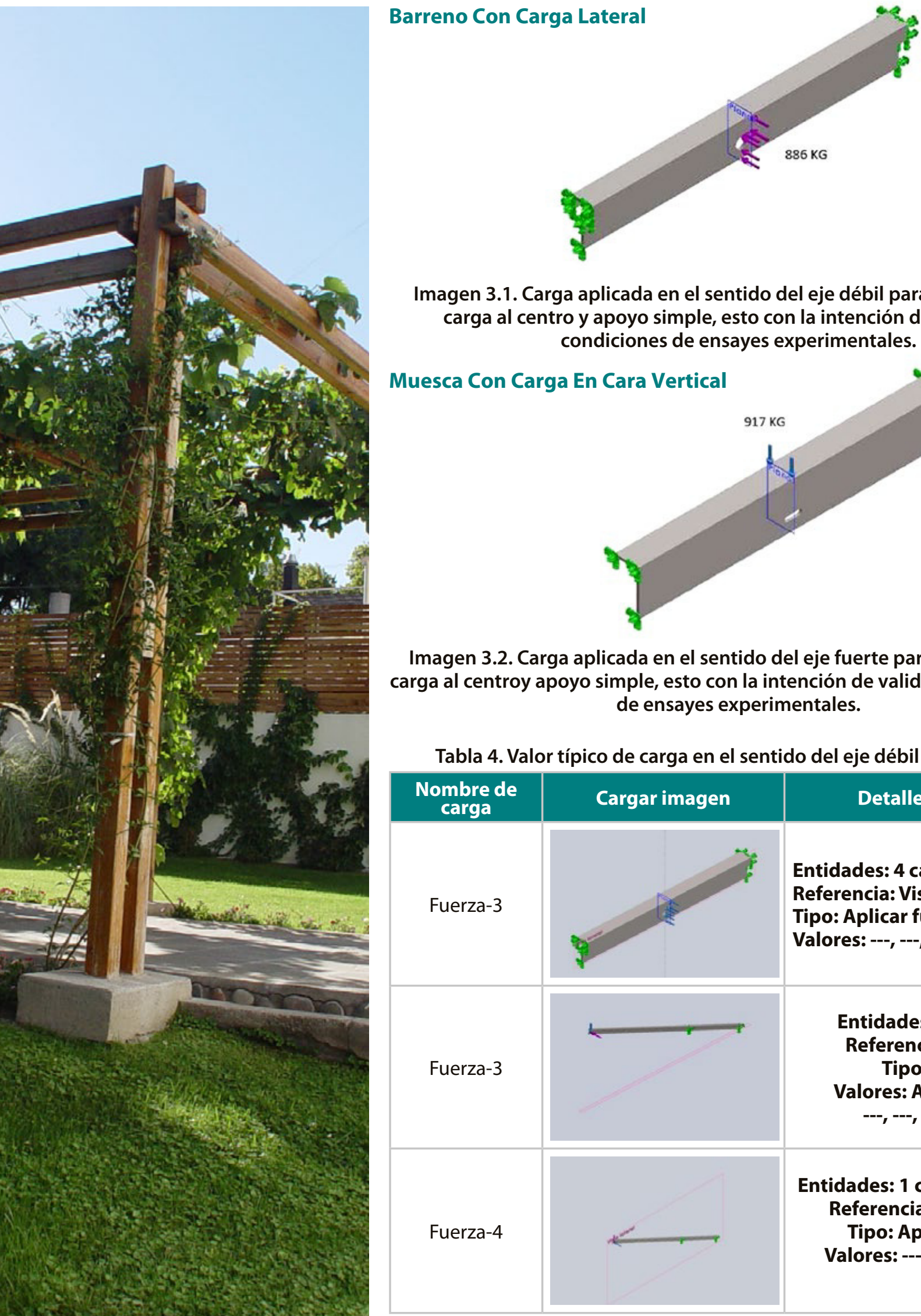

Imagen 3.1. Carga aplicada en el sentido del eje débil para elementos con carga al centro y apoyo simple, esto con la intención de validar con condiciones de ensayes experimentales.

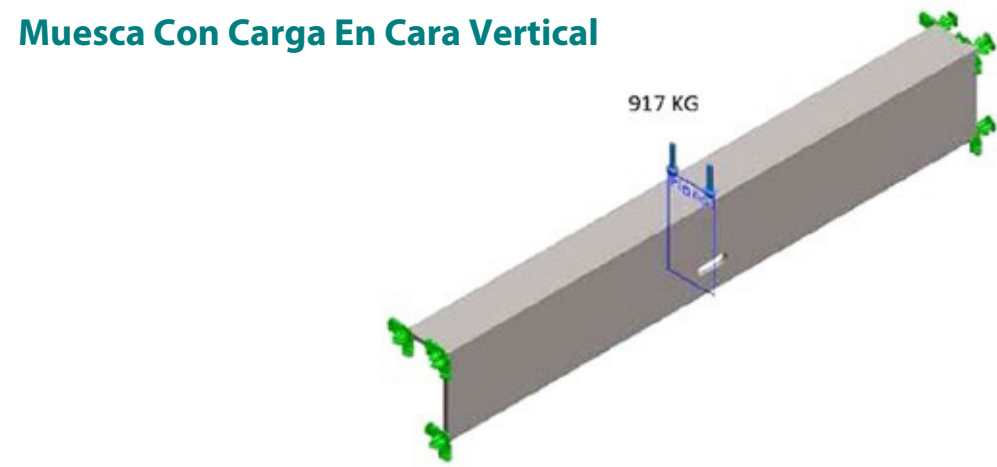

Imagen 3.2. Carga aplicada en el sentido del eje fuerte para elementos con carga al centroy apoyo simple, esto con la intención de validar con condiciones de ensayes experimentales.

Tabla 4. Valor típico de carga en el sentido del eje débil de la sección.

\begin{tabular}{|c|c|c|}
\hline $\begin{array}{c}\text { Nombre de } \\
\text { carga }\end{array}$ & Cargar imagen & Detalles de carga \\
\hline Fuerza-3 & & $\begin{array}{l}\text { Entidades: } 4 \text { cara(s), } 1 \text { plano(s) } \\
\text { Referencia: Vista lateral } \\
\text { Tipo: Aplicar fuerza } \\
\text { Valores: ---, ---, -8828 N }\end{array}$ \\
\hline Fuerza-3 & & $\begin{array}{c}\text { Entidades: } 1 \text { cara(s), } 1 \\
\text { Referencia: plano(s) } \\
\text { Tipo: Planta } \\
\text { Valores: Aplicar fuerza } \\
---,---,-421.83 \mathrm{~N}\end{array}$ \\
\hline Fuerza-4 & $\leftarrow$ & $\begin{array}{c}\text { Entidades: } 1 \text { cara(s), } 1 \text { plano(s) } \\
\text { Referencia: Vista lateral } \\
\text { Tipo: Aplicar fuerza } \\
\text { Valores: ---, ---, -421.83 N }\end{array}$ \\
\hline
\end{tabular}


A continuación se muestran las cargas aplicadas en los modelos de barras.
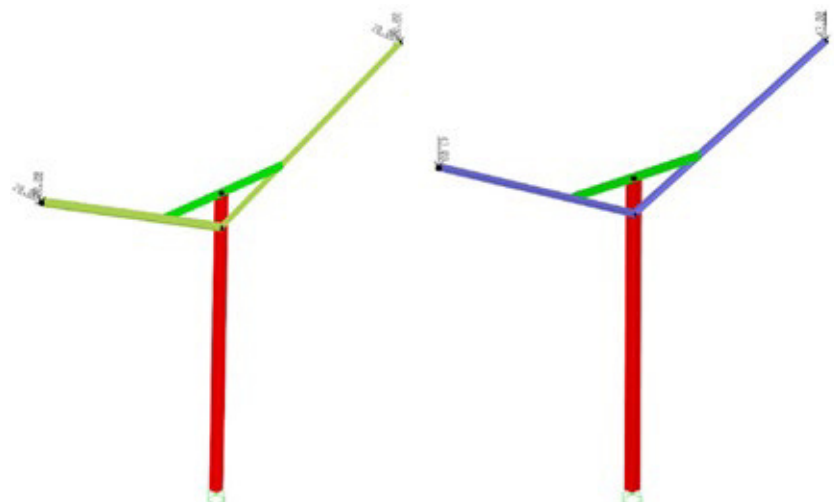

Imagen 3.3. Valor típico de carga gravitacional (43 kgf) en arreglo completo de pérgola. Y Valor típico de carga gravitacionaly lateral simultaneas (20 kgf) en arreglo completo de pérgola.

\section{RESULTADOS}

A continuación se muestran resultados de los ensayes experimentales para los cuales su protocolo de carga fue monótono a la falla con una velocidad constante para todos los especímenes de $40 \mathrm{~kg} / \mathrm{s}$, con apoyo simple con longitud entre apoyos de $305 \mathrm{~mm}$ con dos posiciones del perfil para su aplicación de carga (eje fuerte y eje débil) y tres condiciones de reducción de la sección transversal para cada sección, siendo estas, sin perforaciones, con muesca y con barreno, las cuales se situaron al centro, es decir, justo debajo del punto de aplicación de carga esto para inducir la falla en el punto crítico y observar su comportamiento.

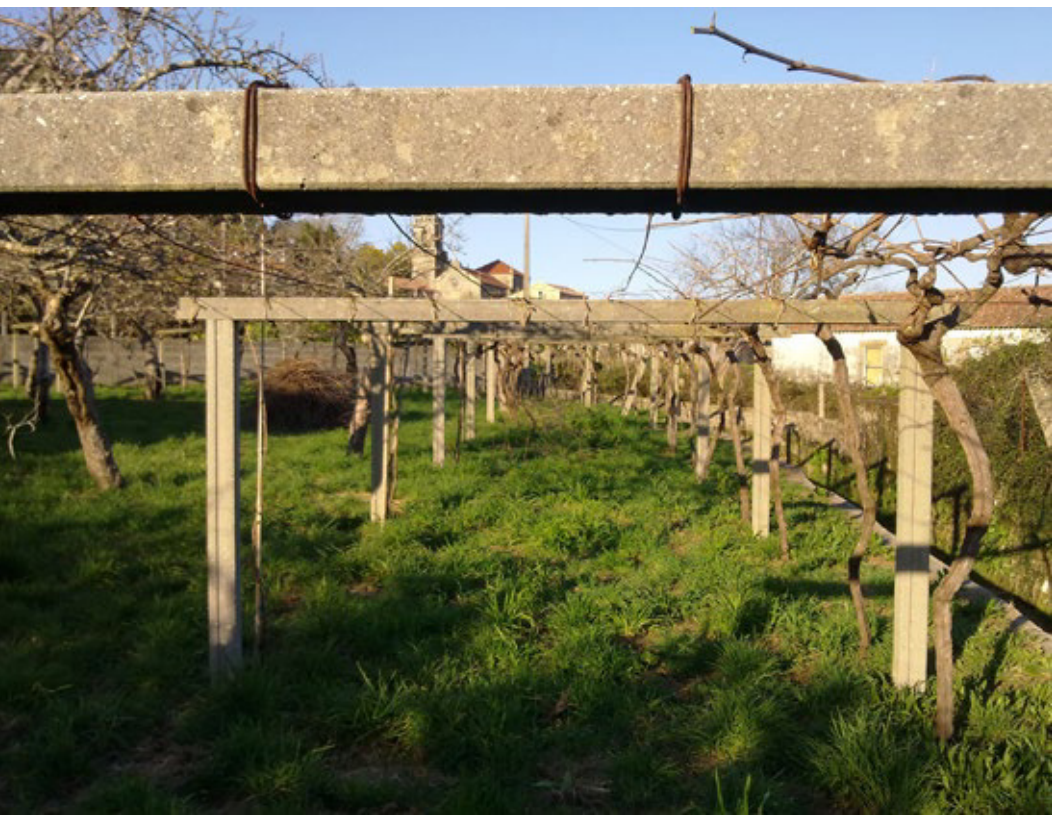

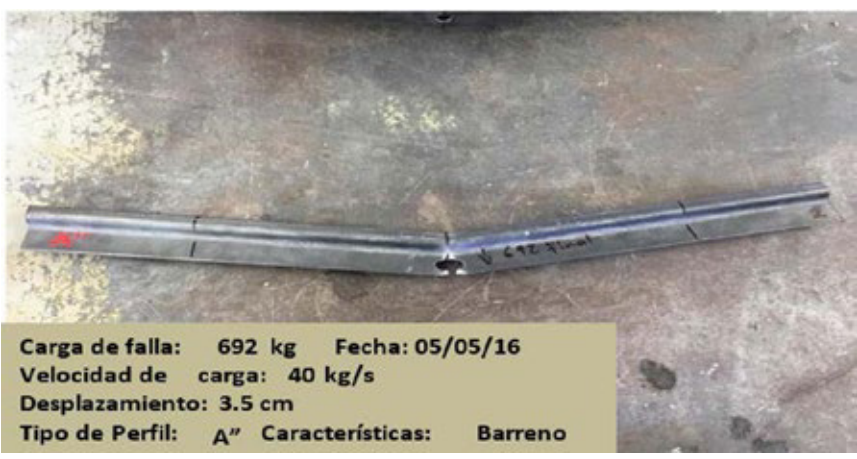

Imagen 4.1. Muestra de ensaye en Perfil tipo A" Barreno

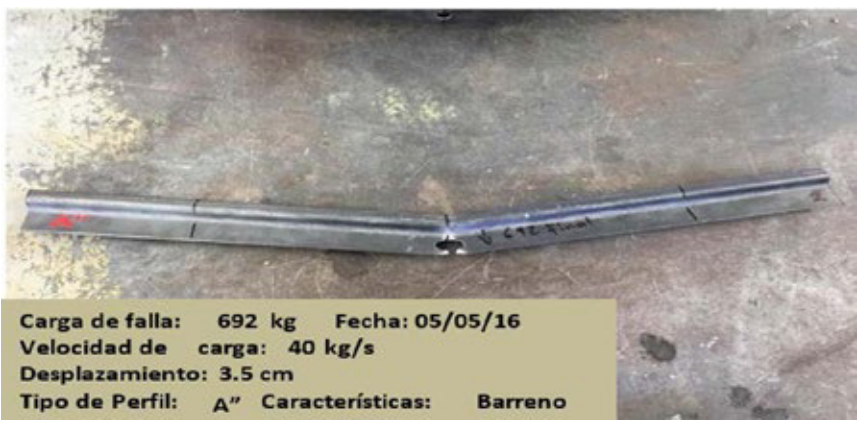

Imagen 4.2. Muestra de ensaye en Perfil tipo A Muesca.

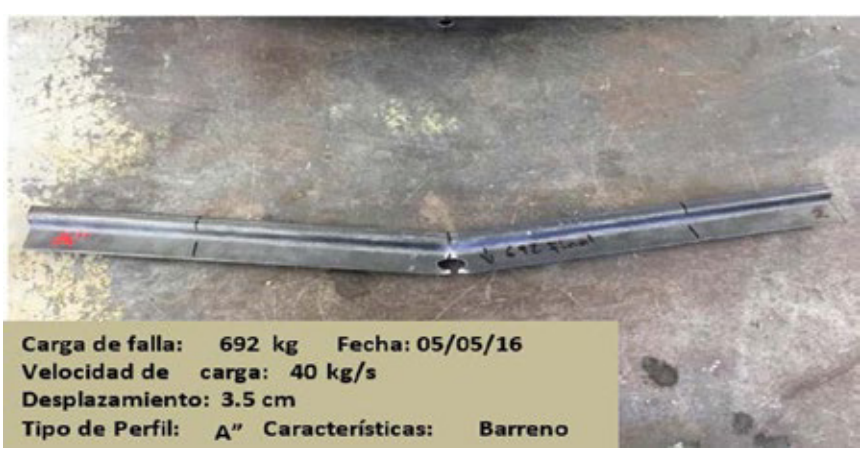

Imagen 4.3. Muestra de ensaye en Perfil tipo L Liso.

\section{CONCLUSIONES}

El análisis y propuesta de perfil componente del sistema de pérgola para soporte de viñedos, sustenta los resultados en cuatro puntos de análisis los cuales consisten en modelado por método de elementos finitos del arreglo general en barras (MEF BARRA), modelado por método de elementos finitos del elemento en solido con condiciones de apoyo reales y condiciones de apoyo de ensaye por flexión (MEF SOLIDO A y MEF SOLIDO S) y por ultimo para validar modelos numéricos resultados de ensaye experimental a flexión.

Por otra parte, las comparativas de perfiles propuestas toman en cuenta la condición más crítica en la sección transversal, es decir, aquella que la reduce por medio de perforaciones, teniendo sección con barreno, con muesca (orificio alargado y corte) y sección sin perforaciones.

Además de ensayar los perfiles en su eje fuerte sobre el cual actuaran las cargas gravitacionales y de uso común, 
como también en su eje débil sobre el cual actuarán cargas extraordinarias accidentales, como por ejemplo viento.

A continuación, se resume el comportamiento de los perfiles y sus condiciones críticas con respecto a los cuatro puntos antes mencionados.

\section{Ensaye experimental a flexión simple (apoyo simple con carga puntual al centro)}

Tal como se puede observar en los gráficos en cuanto a la capacidad de carga a la falla en el eje fuerte es muy similar para los tres perfiles, sin embargo, es importante recalcar que las condiciones de apoyo en la pérgola son distintas y el estado limite gobernante es pandeo lateral inelástico, es decir, que juega un papel muy importante el eje débil del perfil pues entre mayor sea su capacidad mayor resistencia tendrá y podrá desarrollar mejor su capacidad plena. En cuanto a su capacidad a flexión en el eje débil el perfil A" exhibió sin duda un mejor comportamiento que los otros dos perfiles.

Ensaye de perfiles a flexion en eje debil. (lateral)

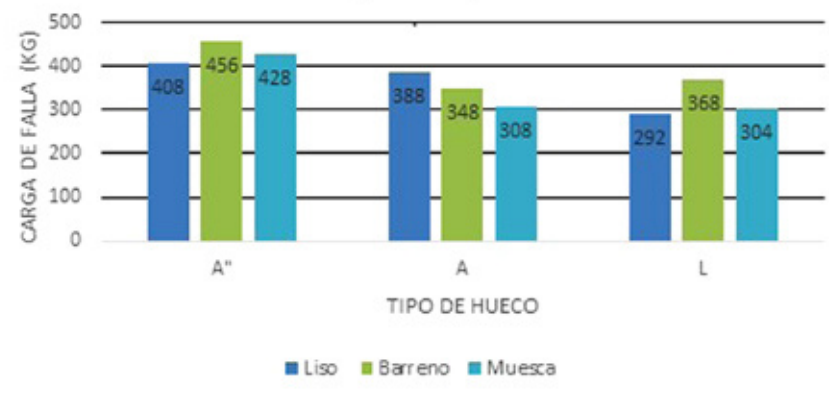

Tabla 6. Ensaye de perfiles eje débil (Carga).

En términos de las deformaciones exhibidas a la falla en el eje fuerte se presentan deformaciones muy similares, sin embargo, en el eje débil también es muy marcada la diferencia del perfil $A^{\prime \prime}$ con respecto a los otros dos, pues muestra un comportamiento más rígido deformándose mucho menos para un mayor nivel de carga.

Ensaye de perfiles a flexion en eje debil. (lateral)

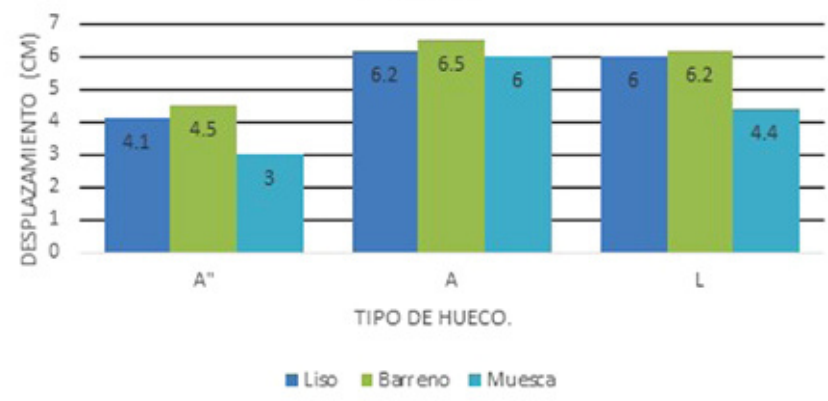

Tabla 6.1. Ensaye de perfiles eje débil (Desplazamiento).

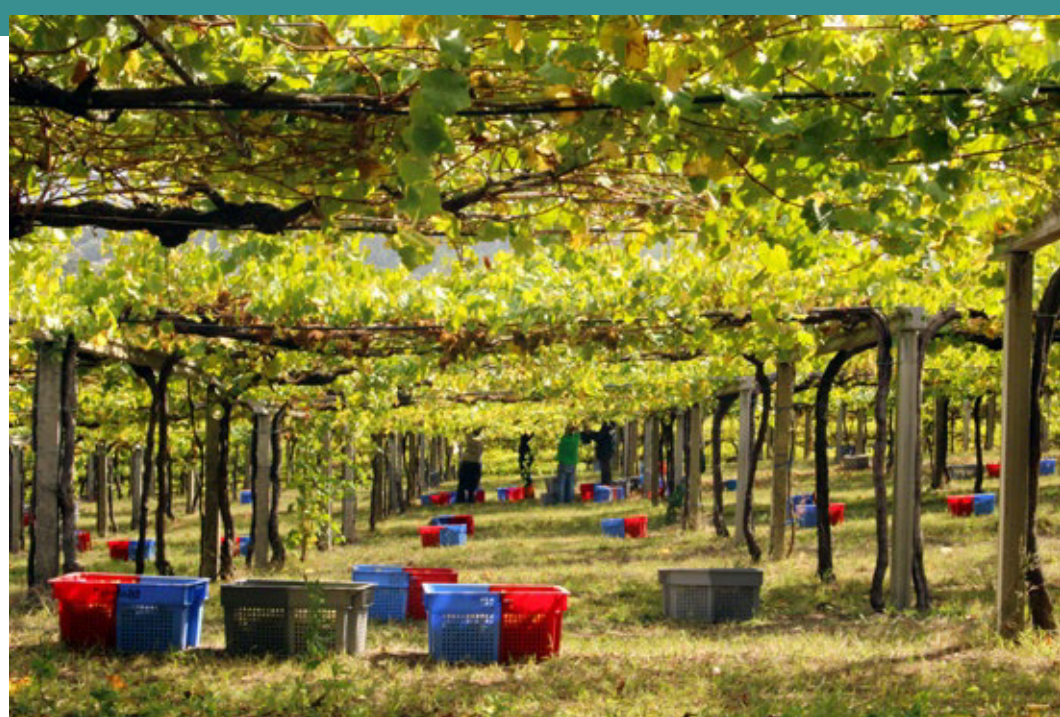

\section{Modelo de Análisis Solido}

Se observa que el perfil $A^{\prime \prime}$ desarrolla un menor esfuerzo para carga vertical esto significa que muestra mayor resistencia ante la carga aplicada, y exhibe también mayor rigidez a flexión esto lo podemos corroborar en el nivel de desplazamiento en el cual es mucho menor que los otros perfiles.

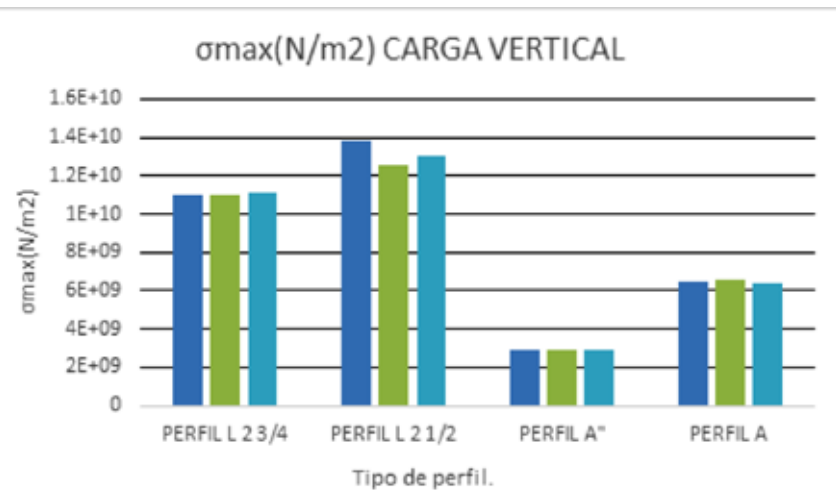

Tabla 6.2. omax Carga Vertical por tipo de perfil.

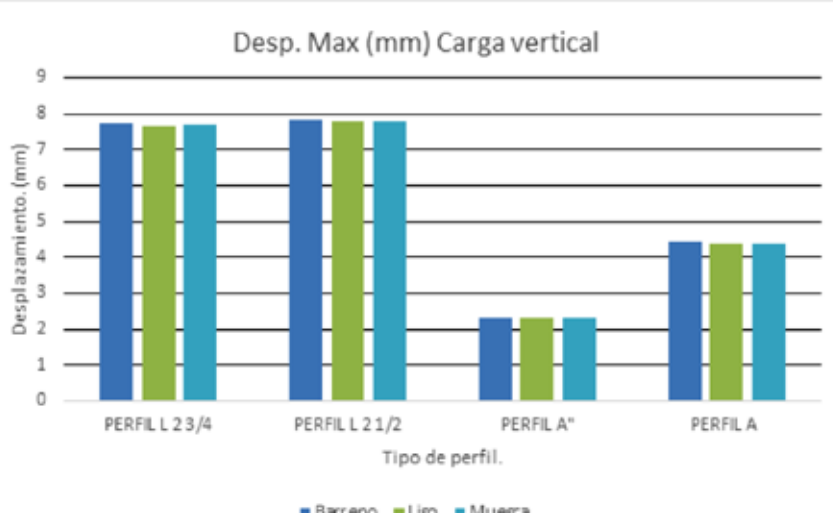

Tabla 6.3. Desplazamiento máximo por tipo de perfil.

En el modelo de sólido en el cual simula de manera aproximada las condiciones de apoyo reales de la pérgola, se observa que los porcentajes de eficiencia, basados en 
el comportamiento obtenido mediante los esfuerzos y desplazamientos máximos, tomando en cuenta el perfil $A^{\prime \prime}$ como referencia, tiene un desempeño estructural satisfactorio con respecto a los demás perfiles modelados.

Porcentaje de eficiencia.

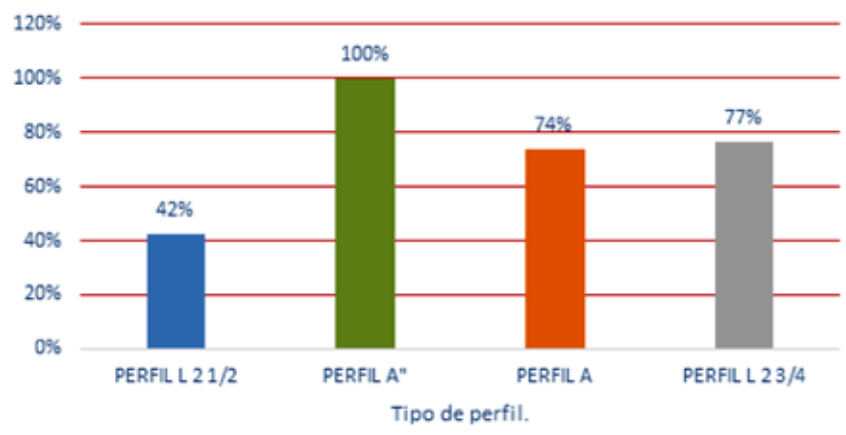

Tabla 6.4. Porcentaje de Eficiencia según el tipo de perfil.

\section{Modelo de Análisis de barras}

En cuanto a los resultados obtenidos en el modelo de barras, para el cual se analizaron además para el perfil $A^{\prime \prime}$ los calibres 13 y 14, en términos de deformaciones tanto para el modelo realizado ante carga gravitacional, así como el de cargas gravitacional y lateral simultáneas, se logra observar que el valor obtenido es menor que el de los otros perfiles, por lo cual, se con este modelo de análisis se refuerza lo obtenido en los anteriores, que el perfil A" exhibe un mejor comportamiento estructural, comportándose más rígido a flexión.

\begin{tabular}{|c|c|c|c|c|c|}
\hline \multicolumn{5}{|c|}{ Eficiencias de los perfiles con respecto a la deformacion(solo carga gravitacional) } & \multirow{2}{*}{$\begin{array}{l}\text { Relacion de def. entre } \\
\text { Ejes } X Y Y\end{array}$} \\
\hline Perril & Def. EjeX & Def. EjeY & Efficiencia-Eje X(j) & Eficiencia-Eje $Y\left(S_{S}\right)$ & \\
\hline $41.75^{\prime \prime} \times 1^{\prime \prime}$ & -1.7341 & .1 .38 & & & 1.257 \\
\hline Tipo A & -0.4428 & -1.2252 & 3.9 & 1.1 & 0.361 \\
\hline Tipo A" UNISON & -0.0291 & -1.6633 & 59.6 & 0.8 & 0.017 \\
\hline Tipo A"Mod. Cal.12 & .0 .0175 & .1 .2577 & 99.1 & 1.1 & 0.014 \\
\hline Tipo A" Mod. Cal.13 & -0.0228 & -1.4448 & 76.1 & 1.0 & 0.016 \\
\hline Tipo A" Mod. Cal.14 & 0.0297 & .1 .7064 & .58 .4 & 0.8 & -0.017 \\
\hline
\end{tabular}

Tabla 6.5. Eficiencias de los perfiles con respecto a la deformación (Carga Gravitacional).

\begin{tabular}{|c|c|c|c|c|c|}
\hline \multicolumn{5}{|c|}{ Eficiencias de los perfiles con respecto a is deformacion(carga gravitacional y lateral) } & \multirow{2}{*}{$\begin{array}{l}\text { Relacion de def. entre } \\
\text { Ejes } X y Y\end{array}$} \\
\hline Perfil & Def. EjeX & Def. EjeY Y & Eficiencia-Eje X(Y) & Eficiencia-Eje $Y(S)$ & \\
\hline $11.75^{\prime \prime} \times 1 "$ & .11 .9013 & .3 .0537 & & & 0.257 \\
\hline Tipo A & .11 .756 & .1 .6518 & 1.0 & 1.8 & 0.141 \\
\hline TIPO A" UNISON & .9 .5266 & .1 .9611 & 1.2 & 1.6 & 0.206 \\
\hline Tipo A" Mod. Cal.12 & .10 .0932 & .1 .2406 & 1.2 & 2.5 & 0.123 \\
\hline Tipo A" Mod. Cal.13 & .11 .33 & .1 .4226 & 1.1 & 2.1 & 0.126 \\
\hline Tipo A" Mod.Cal.14 & .13 .0422 & .1 .6774 & 0.9 & 1.8 & 0.129 \\
\hline
\end{tabular}

Tabla 6.6. Eficiencias de los perfiles con respecto a la deformación (Carga Gravitacional y lateral).

Por lo anteriormente expuesto se recomienda el uso del perfil A", debido a las cualidades estructurales exhibidas.

\section{BIBLIOGRAFÍA}

[1] American Institute of Steel Construction. (2016). ANSI/AISC 360-16 Specification for Structural Steel Buildings. Chicago, Illinois: 130 East Randolph Street.

[2] ASTM International. (2013). E8/E8M Tension Testing of Metallic Materials. United States.

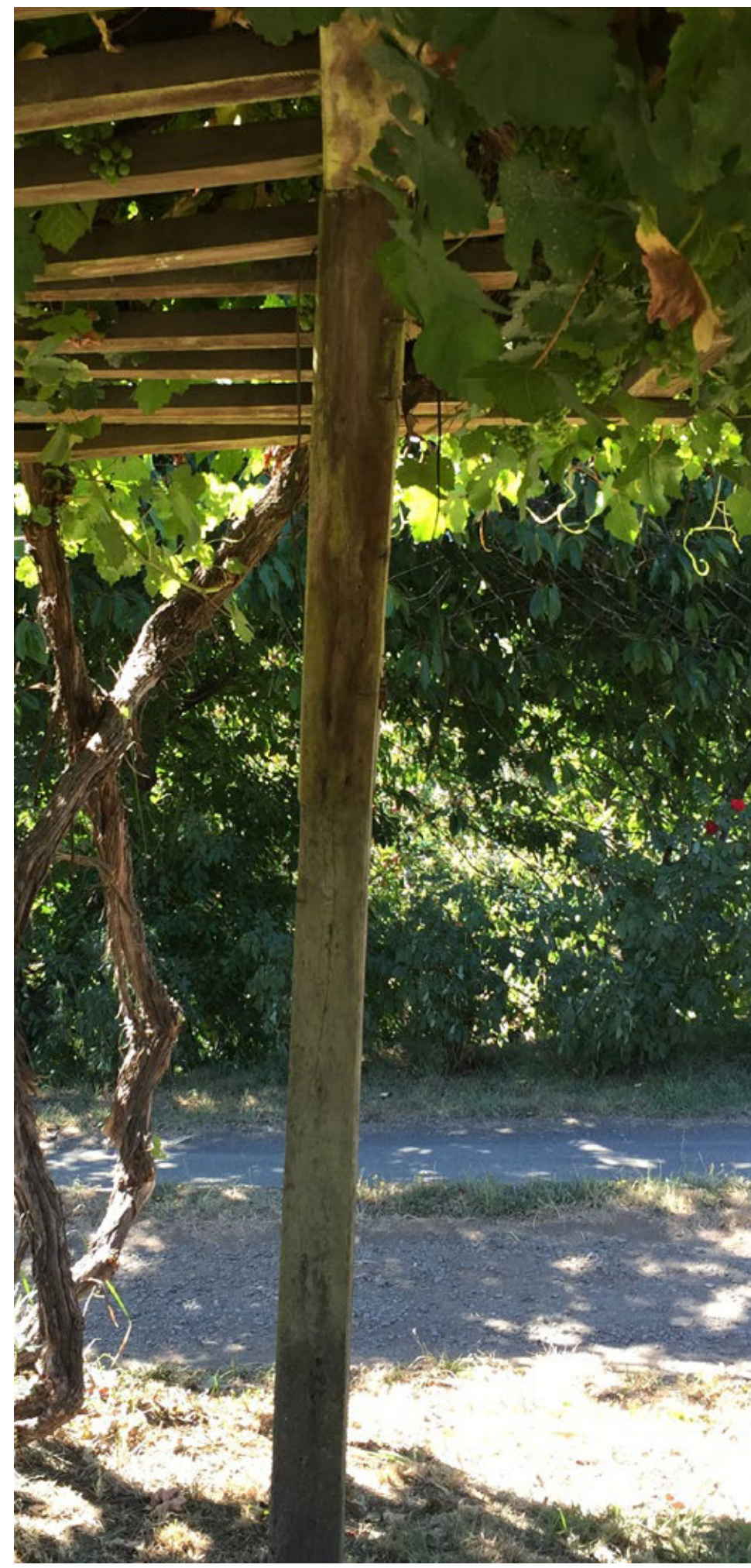

